\title{
Light Scattering: The Angular Position of the Minimum for the $H_{\mathrm{h}}$ Component
}

\author{
J. C. Ravey and P. Mazeron \\ Universite de Nancy I Laboratoire de Biophysique, Centre de ler Cycle, \\ Case Officielle $n^{\circ}$ 140, 54037 NANCY CEDEX, France.
}

(Received July 27, 1973)

\begin{abstract}
A modified photogoniodiffusometer has been used for the accurate determination of the minimum intensity for the $H_{\mathrm{h}}$ component of the light scattered from various suspensions. The angular position $\theta_{\mathrm{m}}$ of this minimum depends both on the shape and on the optical anisotropy of the scattering particles. A few examples are presented showing that the theoretical relations are correct.
\end{abstract}

KEY WORDS Light Scattering / Optical Anisotropy / Shape of Particle /

A series of theoretical papers has recently been devoted to light scattering by anisotropic particles $(1,2,3)$. Using Rayleigh-Debye's approximation, it has been shown theoretically that the minimum value of the $H_{\mathrm{h}}$ component of the intensity of the light scattered from various suspensions occurs at scattering angles $\theta_{\mathrm{m}}$ less than $\pi / 2$, when the product $\delta \omega$ was positive and vice versa. ${ }^{1}$ Here $\delta$ is the optical anisotropy of the scattering particles and $\omega=p^{2}-1$, where $p$ is the ellipticity of the particle $(0 \leq p \leq \infty)$. The theoretical expressions giving the $\theta_{\mathrm{m}}$ value as a function of $\delta$ for various scattering particle shapes are rather complicated. Let us give two interesting asymptotic cases:

For large discs, the $\theta_{\mathrm{m}}$ value can be determined from the following relation, for any values of $\delta$ :

$$
\sin ^{2} \frac{\theta_{\mathrm{m}}}{2}=\frac{(1+2 \delta)}{(2+\delta)}
$$

For large rod shaped particles, the anisotropy of which is small, one obtains:

$$
\sin ^{2} \frac{\theta_{\mathrm{m}}}{2} \simeq \frac{1}{2}\left(1-\frac{3}{4} \delta\right)
$$

The case of absorbing particles (graphite suspensions in alcohol) is discussed by means of the Physical Optics Approximation. ${ }^{4}$

The purpose of this paper is to investigate whether the experimental dependence of the minimum for the $H_{\mathrm{h}}$ component scattered by particles of various shapes and anisotropies agrees with the theory developped within the Rayleigh-Gans-Debye approximation $(1,2,3)$. Such original investigations imply rather precise light-scattering measurements which we have been able to perform with our modified photometer.

\section{RESULTS AND DISCUSSION}

The modified photogoniodiffusometer used for our measurements has been previously described. ${ }^{5}$ Key points are that the method is based on the use of a lock-in amplifier and on dynamic measurements of modulated intensities, the values of which are recorded on a galvanometer.

Tobacco Mosaic Virus (TMV)

Let $L$ be the length of the TMV rod, $\lambda$ the wavelength of the green light in the medium. We have found by a classical light scattering experiment on aqueous suspensions of TMV that $L / \lambda \simeq 0.70$.

The TMV particle is kown to have an optical anisotropy $\delta$ about a few percent: If $g_{1}$ and $g_{2}$ are its principal optical polarizabilities per unit volume, thus

$$
\delta=\frac{g_{1}-g_{2}}{g_{1}+2 g_{2}}
$$

From electric birefringence measurements, some values of $\left(g_{1}-g_{2}\right)$ have been obtained:

$$
g_{1}-g_{2}=8.9 \times 10^{-3}, \quad O^{\prime} \text { Konski and Haltner }{ }^{10}
$$




$$
\begin{array}{ll}
g_{1}-g_{2}=6.1 \times 10^{-3}, & \text { O'Konski, et al. }{ }^{11} \\
g_{1}-g_{2}=9.0 \times 10^{-3}, & \text { Stoylov, et al. }{ }^{12} .
\end{array}
$$

From the following quantities:

$$
\begin{aligned}
& \bar{v}=0.74 \text { partial specific volume of the TMV } \\
& \text { particle }
\end{aligned}
$$

$\mathrm{d} n / \mathrm{d} c=0.184$ its refractive index increment

$n_{\mathrm{s}}=1.33$ the refraction index of the solvent (water) and using the well-known relation:

$$
\frac{\mathrm{d} n}{\mathrm{~d} c}=\frac{2 \pi \bar{v}}{n_{\mathrm{s}}} \frac{g_{1}+2 g_{2}}{3}
$$

one gets $\delta$ equal to $5.6 \times 10^{-2}, 3.9 \times 10^{-2}$, and $5.7 \times 10^{-2}$ respectively. These $\delta$ values agree with other determinations:

$\delta=4.4 \times 10^{-2}$ (light scattering in an electric field, Premilat and Mazeron ${ }^{6}$ )

$\delta=5.0 \times 10^{-2}$ (measurements of depolarization ratio, this work)

$\delta=3.6 \times 10^{-2}$ (Kerr effect, Wippler $\left.{ }^{13}\right)$.

We note that for rod-shaped particles (ellipticities $p=20$ ) the intrinsic anisotropy of which is zero, the theoretical quantity $g_{1}-g_{2}$, calculated

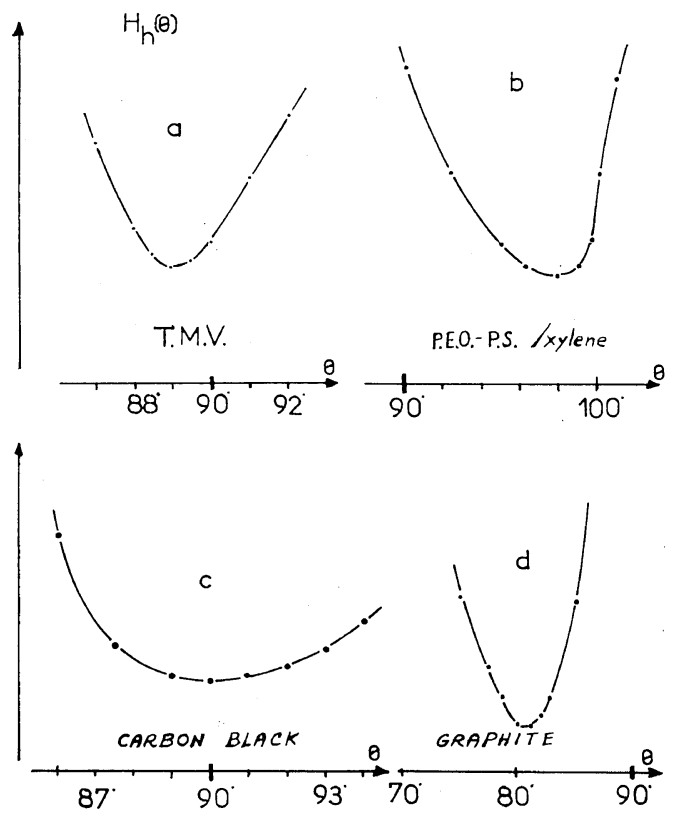

Figure 1. Experimental variation in the $H_{\mathrm{h}}$ component of the intensity of the light scattered from various suspensions.

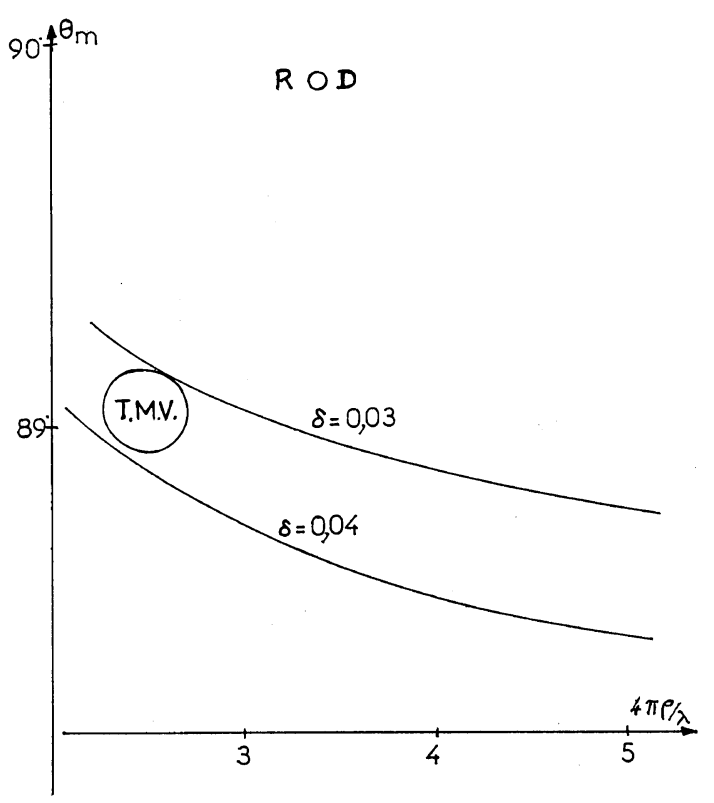

Figure 2. Theoretical (solid line) and experimental (circle) $\theta_{\mathrm{m}}$ values for rod as a function of $4 \pi \rho / \lambda$ ( $\rho$, radius of gyration of the rod).

by Maxwell formula is $\simeq 5 \times 10^{-3}\left(n_{\mathrm{s}}=1.35\right)$, i.e., $\delta \simeq 3.2 \times 10^{-2}$. For such a value, it is expected to have $\theta_{\mathrm{m}}$ close to $89^{\circ}$. It can be seen on Figure 1 (curve a) and Figure 2 that this is the experimental value which is determined within a precision better than $0.5^{\circ}$. It has been ascertained by rotating the scattering cell about a vertical axis that eventual optical defects do not modify the $\theta_{\mathrm{m}}$ value found. The agreement between the theoretical and measured value is quite satisfactory.

Copolymer PEO-PS Single Crystals

Poly(ethylene oxide)-polystyrene single crystals of various sizes $(0.65 \mu, 2 \mu, 5 \mu)$ were prepared by B. Lotz (Centre de Recherches sur les Macromolecules, Strasbourg) in paraxylene. ${ }^{7}$ For such disc-like particles, the asymptotic relation $\mathrm{A}$ is valid. The minimum for the $H_{\mathrm{h}}$ component scattered by these suspensions takes place for $\theta_{\mathrm{m}}=98^{\circ}$. (Figure 1, curve b) and depends to some extent on the concentration and size of the scattering particles. Such a value of $\theta_{\mathrm{m}}$. leads to a positive optical anisotropy $\delta$

$$
\delta=+0.11
$$


Light Scattering: The Angular Position of the Minimum for the $H_{\mathrm{h}}$ Component

as expected; for comparison the value found by Picot, et al., ${ }^{8}$ by means of depolarization measurements for $\theta=0$, was $\delta=+0.13$.

\section{Carbon Blacks}

The optical anisotropy of carbon blacks in cyclohexanone is less than 0.015 . As the anisodiametry is not important, the product $\delta \omega$ is very close to zero, so that $\theta_{\mathrm{m}} \simeq \pi / 2$. It has been found experimentally that $\theta_{\mathrm{m}}=\pi / 2$ (Figure 1 , curve c). Thus this measurement is a test for the good optical adjustment of the apparatus.

\section{Graphite Suspensions}

The graphite suspensions Dag S 386 (colloidal graphite in alcohol) have been supplied by Acheson (Scheemda-Holland). The mean particle diameter measured by electron microscopy is about $2 \mu$.

For suspensions of such small particles of graphite, Rayleigh-Debye approximation evidently does not apply. By using the physical Optics Method which approximates the boundary conditions of the wave equation, ${ }^{4}$ Ravey ${ }^{9}$ has calculated the $H_{\mathrm{h}}$ component of the intensity "scattered" by these absorbing and reflecting lamellar particles. In this approximation, we suppose that the spherical waves emitted from all the points of the particle surface interfere and we calculate the resulting amplitude and polarization of the far field scattered wave. Both of these quantities depend on the polarization and incidence angle of the incident wave.

${ }^{*}$ For sufficiently large scattering areas, as a result of the interferences, only the wave reflected in the direction of specular reflection remains. Such large particles reflect the light in the $\theta$ direction of observation of the photometer only if they are vertically oriented with an incident angle $i=(\pi-\theta) / 2$.

For the $H_{\mathrm{h}}$ component, the electric fields of the incident and reflected waves lie then in the plane of incidence of these large particles; as a result $H_{\mathrm{h}}$ should be proportional to $\left|R^{+}\right|^{2}$ where

$$
R^{+}=\frac{n^{2} \cos i-\sqrt{n^{2}-\sin ^{2} i}}{n^{2} \cos i+\sqrt{n^{2}-\sin ^{2} i}}
$$

which should attain a minimum for some value of $i$ referred to as the pseudo-Brewster angle.

${ }^{*}$ Conversely, the scattered intensities have to be calculated at any given orientation of smaller disc-like particles.

Subsequently, an integration over all the orientations of these particles is effected by means of numerical computer calculations. For graphite suspenssions in ethyl alcohol, the complex refractive index of which is $n$

$$
n=1.6-0.82 i
$$

The theoretical results are the following:

\begin{tabular}{cc}
\hline Diameter of the particle & $\theta_{\mathrm{m}}$ \\
\hline $1 \mu$ & $83^{\circ}$ \\
$2 \mu$ & $78^{\circ}$ \\
\hline
\end{tabular}

The experimental result for the above value of $n$ is about $\theta_{\mathrm{m}}=80^{\circ}$. (Figure 2, curve d), whereas a pseudo-Brewster angle should be close to $58^{\circ}$ for very large particles of graphite in in alcohol suspensions, and should correspond to a minimum for $H_{\mathrm{h}}$ at $\theta=64^{\circ}$. This angular shift is in good agreement with the theoretical value obtained for discs of a diameter of about $2 \mu$.

\section{CONCLUSION}

In conclusion, the method seems accurate enough to offer a powerful tool for studying either the optical anisotropy or the shape of scattering particles, and may also be suitable for measuring optical anisotropy changes or shape changes which would occur in a solution.

\section{REFERENCES}

1. J. C. Ravey, Europ. Polym. J., 8, 937 (1972).

2. J. C. Ravey, ibid., 9, 47 (1973).

3. J. C. Ravey, ibid., 9, 57 (1973).

4. P. Beckmann, in "The Depolarization of Electromagnetic Waves", the Golem Press, 1968, p 76.

5. J. C. Ravey, P. Mazeron, and Y. Sere, Polymer, 15, 77 (1974).

6. S. Premilat and P. Mazeron, J. Chim. Phys., 68, 6, 954 (1971).

7. B. Lotz, A. J. Kovacs, G. A. Basset, and A. Keller, Kolloid-Z.Z. Polym., 209, 115 (1966).

8. C. Picot, G. Weill, and H. Benoit, J. Colloid Interfac. Sci., 27, 3 (1968).

9. J. C. Ravey, unpublished results. 
10. C. T. O'Konski and A. J. Haltner, J. Amer. Chem. Soc., 79, 5634 (1957).

11. C. T. O'Konski, K. Yoshioka, and W. H. Orttung, J. Phys. Chem., 63, 1558 (1959).
12. S. Stoylov, Y. Mauss, C. Hornick, and G. Weill, Izw. Otd. Khim. Nauki, Bulgar. Akad. Nauk, 1, $\mathrm{n}^{\circ}$ 4, 99 (1968).

13. C. Wippler, These, Univ. de Strasbourg, 1955. 